\title{
A Novel Control Method of Dynamic Filter for Composite Power Load
} Haiping LIN ${ }^{1, a}$, Jing CHEN ${ }^{2, b}$, Shijie DENG ${ }^{2, c}$

${ }^{1}$ Department of Computer, Wenzhou Vocational and Technical College ,Wenzhou, 325000, China

${ }^{2}$ School of Automation, Wuhan University of Technology, Wuhan, 430070, China

aemail: LHPXYPA@126.com, bemail: jingchen680@163.com, cemail: dengsj91@qq.com

Keywords: Harmonic filtering, Control Method, Dynamic Filter

\begin{abstract}
The working compound electrical loads are characterized by a large number of harmonics. It may pull down the voltage of the power supply network and influence the other electrical devices. Therefore, a control method of dynamic filter for the composite power load was developed. In this paper, a controller is designed to control the dynamic filter to keep the harmonics in permitted ranges by harmonic filtering. The following works have been done in the study: harmonic analysis for the composite power load; the theory of the control method of dynamic filter for the composite power load; the implementation of the control method of dynamic filter for the composite power load. It can improve operation efficiency when the load is working, save energy, stabilize the voltage of the power supply network.
\end{abstract}

\section{Introduction}

With the rapid development of the national economy, the number of the nonlinear loads and devices are used in all industry fields. The harmonics generated by nonlinear load and device has been declined power quality. At the same time, the harmonics bring the transformer and motor overheating, the center line overloading, the relay error acting, electronic control system failure, telephone interference and so on[1]. So the harmonic suppression is imperative. It can improve power quality and energy saving. Therefore, the generation, transmission, control and suppression of harmonic current in the power system is paid great attention by academia, industry and government departments. Domestic and foreign scholars and engineering and technical personnel are researching on the harmonic filtering technology and device.

In the past 5 years, the author researched the dynamic harmonic filtering technology based on power electronic reactor, and the developed of the passive dynamic harmonic filter[2] [3] [4] [5]. It has been found that the harmonics generated by the composite power load has different characteristics. The key technology is the automatic tuning method. It can automatic tune to keep the harmonics in permitted ranges when the harmonics is changing.

In this paper, based on the previous research, a control method of dynamic filter for the composite power load was developed. The following works have been done in the study: analyze harmonic characteristics of the composite power load; principle of the control method of dynamic filter for the composite power load; implementation of the control method of dynamic filter for the composite power load. It can improve operation efficiency when the load is working, save energy, stabilize the voltage of the power supply network.

\section{Analyze Harmonic Characteristics of Composite Power Load}

In industrial field, the harmonics produced by the composite power load, such as medium frequency furnace, changes in different time periods. With the use of the composite power load, the harms of the power network harmonic become more and more serious. When the composite power load runs at full-load, the amount of the harmonic is maximum value $\left(\mathrm{I}_{\mathrm{M}}\right)$. When the composite power load runs at no-load, the amount of the harmonic is the minimum value $\left(\mathrm{I}_{\mathrm{N}}\right)$. The schematic diagram of the harmonic characteristic curve is shown in the Fig.1. 


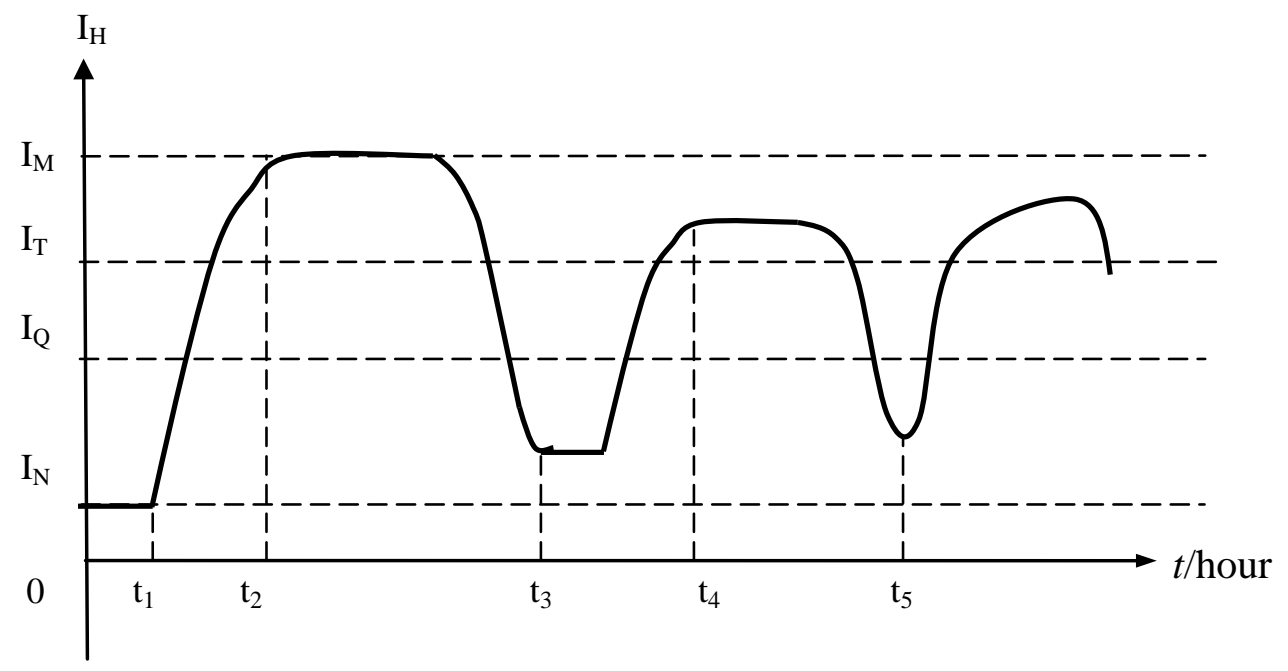

Fig 1. The schematic diagram of the harmonic characteristic curve

In Fig $1, \mathrm{I}_{\mathrm{N}}$ is the lower limit of the harmonic current, $\mathrm{I}_{\mathrm{M}}$ is the upper limit of the harmonic current. $\mathrm{I}_{\mathrm{T}}$ is $(0.7-0.8)$ times of $\mathrm{I}_{\mathrm{M}}, \mathrm{I}_{\mathrm{Q}}$ is $(0.5-0.6)$ times of $\mathrm{I}_{\mathrm{M}}$.

For different harmonic value at the different running time, the composite power load runs in different state. Running states of the composite power load are shown in Table.1.

Table 1 Running states of the composite power load during a period

\begin{tabular}{ccc}
\hline Time & $\mathrm{I}_{\mathrm{H}} / \mathrm{A}$ & Running states \\
\hline $\mathrm{I}\left(0 \sim \mathrm{t}_{1}\right)$ & 0 & Stop \\
$\mathrm{II}\left(\mathrm{t}_{1} \sim \mathrm{t}_{2}\right)$ & $\mathrm{I}_{\mathrm{N}} \uparrow \mathrm{I}_{\mathrm{M}}$ & Start \\
$\mathrm{III}\left(\mathrm{T}_{2} \sim \mathrm{t}_{3}\right)$ & $\mathrm{I}_{\mathrm{M}}$ & Run \\
$\mathrm{IV}\left(\mathrm{T}_{3} \sim \mathrm{t}_{4}\right)$ & $\mathrm{I}_{\mathrm{M}} \downarrow \mathrm{I}_{\mathrm{N}}$ & unload \\
$\mathrm{V}\left(\mathrm{T}_{4} \sim \mathrm{t}_{5}\right)$ & $\mathrm{I}_{\mathrm{N}}$ & pause \\
\hline
\end{tabular}

\section{Principle of Control Method of Dynamic Filter for Composite Power Load}

The dynamic filter for the composite power load (referred to as the "dynamic filter") is the combination of the power electronics technology, the variable reactance technique and the filtering techniques. The dynamic filter consists of an adjustable capacitor sets, inductance converter, thyristor circuit, intelligent electricity detection module (IEDM), controller, and so on. The topological structure of the dynamic filter is shown in Fig.2.

In Fig. 2, the signal of voltage and current of the composite power load is measured by voltage and current transformers. It is sent to IEDM and converted into digital signals, such as current, voltage, the value and the time of the harmonic current. The frequency of the main harmonic current is recorded as $f_{x}$.

\section{(1) The starting/stopping of the dynamic filter}

When the value of harmonic current reaches to $\mathrm{I}_{\mathrm{T}}$, the dynamic filter should be started. When the value of harmonic current falls to IQ, the dynamic filter should be stopped. It can keep the harmonic current stable and improve the efficiency of the filtering.

\section{(2) The regulation of the dynamic filter}

When the harmonic of the composite power load has changed, according to the principle of passive LC filter, the controller properly regulates the inductance value $\mathrm{L}$ of the inductance converter by the thyristor circuit and capacitance $\mathrm{C}$ by switching on/off the capacitance sets. It 
forms the low impedance circuit with a resonant frequency $f_{y}$. At this time, the frequency $f_{x}$ and $f_{y}$ can satisfy the following formula:

$$
f_{x}=f_{y}
$$

When $f_{x}$ changes, $f_{y}$ also can be adjusted by the controller. So the harmonic can be absorbed effectively.

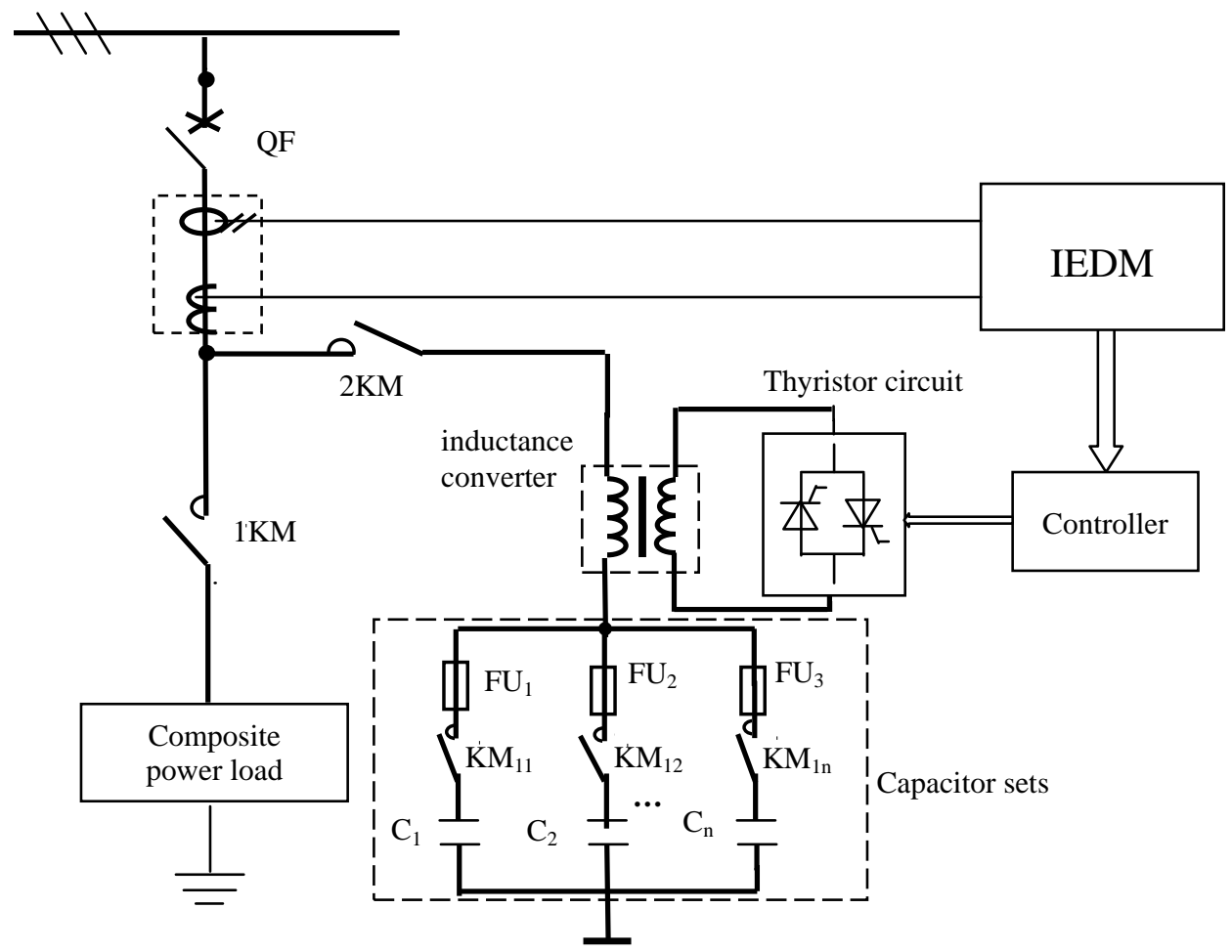

Fig 2. The topological structure of the dynamic filter

\section{Implementation for Control Method of Dynamic Filter for Composite Power Load}

(1) Connecting the power supply, then the intelligent electricity detection module and the controller work;

(2) Setting the parameters of the dynamic filter, such as the maximum harmonic current- $\mathrm{I}_{\mathrm{M}}$, and the minimum harmonic current- $\mathrm{I}_{\mathrm{N}}$, the upper limit $\mathrm{I}_{\mathrm{M}}$ and of the lower limit $\mathrm{I}_{\mathrm{Q}}$ of the harmonic current $\mathrm{I}_{\mathrm{H}}$.

(3) Switching on the contactor $1 \mathrm{KM}$, and the composite power load runs;

(4) Basing on the method for starting/stopping the dynamic filter, when the value of harmonic current reaches $\mathrm{I}_{\mathrm{T}}$, contactor $2 \mathrm{KM}$ can be switched on, and the dynamic filter runs;

(5) Basing on the method for regulating the dynamic filter, the signal of the harmonic can be analyzed by the controller, and then the contactors KM11 KM1n can be switched on/off to get the suitable capacitance $\mathrm{C}$ of the capacitance sets; the controller outputs the pulse signal to adjust the thyristor circuit. The proper inductance value $L$ of the inductance converter is adjusted It forms a low-impedance passive LC filter system and satisfies the formula (1).

\section{Conclusion}

A control method of dynamic filter for the composite power load is advanced in this paper. The following works have been done in the study: analyze harmonic characteristics of the composite power load; principle of the control method of dynamic filter for the composite power load; implementation of the control method of dynamic filter for the composite power load. It can improve operation efficiency when the load is working, save energy, stabilize the voltage of the 
power supply network.

The control method of dynamic filter can improve operation efficiency when the load is working, save energy, stabilize the voltage of the power supply network. It has been be successfully applied to loads such as the intermediate frequency furnace when it is used in the steelmaking.

\section{Acknowledgement}

This work was financially supported by the natural science foundation of Zhejiang Province (Granted No: LY14E070003).

\section{References}

[1] Yuhua Wang. Discussion on The Harmonics Problem and Management for The Low-voltage Power Grid [J]. Electrical Appliance Industry, 2009, (7): 44- 46.

[2] Jing Chen, Min Chen, Youxin Yuan, Chun Xiao, Yu Wang. Design of Control and Drive System of IGBT Based on Dynamic Harmonic Filter. 2011 International Conference on Intelligent Computer Technology and Automation. IEEE Computer Society, 2011: 408-410.

[3] Jing Chen, Lei Lei, Youxin Yuan. Development and application of A New Dynamic Tuned Passive Filter [J]. Electrical Engineering Technology, 2013, 2(3): 3-4.

[4] Haiping Lin, Jing Chen, Siyu Huang, Youxin Yuan. An auto-tuning filtering method based on variable reactors. Lecture Notes in Electrical Engineering, 2014, 238: 859-865

[5] Youxin Yuan, Yiping Xiao. Research on One Kind of Variable Reactor [A]. 2009 International Workshop on Intelligent Systems and Applications[C].Wuhan, 2009, pp. 1847-1850. 